\title{
A neutralização do astigmatismo corneano durante a cirurgia da catarata por meio de lente intraocular tórica: resultados
}

\author{
The neutralization of corneal astigmatism during cataract \\ surgery using toric intraocular lens: results
}

Virgilio Centurion'; Augusto Cézar Lacava'; Juan Carlos Caballero'

\section{ResUMO}

Objetivo: apresentar os resultados refracionais da utilização de lente intra-ocular tórica na correção do astigmatismo corneano durante a cirurgia da catarata. Métodos: Foram estudados prospectivamente 27 olhos de 18 pacientes portadores de catarata com astigmatismo corneano topográfico maior que 1,0DC e menor que 3,0DC, submetidos à cirurgia de catarata pelo sistema de facoemulsificação no período de julho a dezembro de 2007. Resultados: A acuidade visual não corrigida foi de $\geq 20 / 40$ em 92,59\% (25 olhos); a refração pós-operatória foi plana em $66,61 \%$ e entre $\pm 0,50$ em $92,60 \%$. Conclusão: a utilização da LIO tórica se apresenta como uma boa opção para a neutralização do astigmatismo corneano entre 1,0 e 3,0 dioptrias.

Descritores:Lentes intraoculares; Astigmatismo; Extração de catarata; Facoemulsificação; Biometria/métodos; Erros de refração

\footnotetext{
${ }^{1}$ Oftalmologistas do Instituto de Moléstias Oculares - IMO - São Paulo (SP), Brasil;

Trabalho realizado no Instituto de Moléstias Oculares - IMO - São Paulo (SP), Brasil. 


\section{INTRODUÇÃO}

$\mathbf{0}$ grandes avanços na biometria ${ }^{(1,7)}$ permitem que a cirurgia do cristalino com implante de LIO possa alcançar a correção de vícios de refração como miopia, hipermetropia e presbiopia, porém o astigmatismo corneano tem um enfoque diferente.

A correção deste problema refracional pode ser realizado através de: a) incisões relaxantes corneanas ou variando o local e o tamanho da incisão corneana; b) o implante de lente intraocular tórica ${ }^{(8,9)}$.

A frequência do astigmatismo pré-existente em olhos com catarata varia entre $2,0 \%$ a $20,0 \%{ }^{(10-13)}$. A literatura estima que de 15 a $29 \%$ dos olhos com catarata tenham astigmatismo maior que 1,5 dioptrias ${ }^{(12,1,1,1)}$. A incidência de astigmatismo maior que 3,0 dioptrias é menor, em torno de 2 a $3 \%^{(11,16-19)}$.

Atualmente, há disponível no mercado três (3) modelos de lente intra-ocular tórica: Staar Toric IOL (Staar Surgical, Monrovia, California), AcrySofToric IOL (Alcon Laboratories, INC, Fort Worth, Texas), MicroSil IOL ( Human Optics, Erlangen, Germany).

A lente utilizada neste trabalho é a SN60T-Alcon que tem três (3) modelos: SN60T3, T4 e T5 que corrigem astigmatismo de 1,$03 ; 1,55 ; 2,06$ dioptrias no plano corneano $^{(15)}$.

Objetivo do trabalho é verificar a acuidade visual, a previsibilidade e estabilidade refracional e rotacional da LIO tórica na correção do astigmatismo corneano na cirurgia da catarata.

\section{MÉTODOS}

Estudo prospectivo, consecutivo, linear, não comparativo de 27 olhos de 18 pacientes portadores de catarata com astigmatismo corneano topográfico maior que 1.0 DC e menor que 3.0 DC, submetidos à cirurgia de catarata pela técnica da facoemulsificação no período de $07 / 2007$ a 12/2007.

Os critérios de exclusão foram: cirurgia refrativa corneana prévia, astigmatismo assimétrico e/ou irregular, complicações per-cirúrgicas.

Todos os pacientes foram submetidos a exame oftalmológico completo, associado a exames complementares como a biometria óptica (IOL Master - Zeiss), mensuração do poder corneano por meio da ceratoscopia de elevação pelo tomógrafo de segmento anterior (Pentacam - Oculus), fórmula Holladay II, microscopia especular e a oftalmoscopia indireta.

Para o cálculo do modelo da LIO SN60T foi utili-

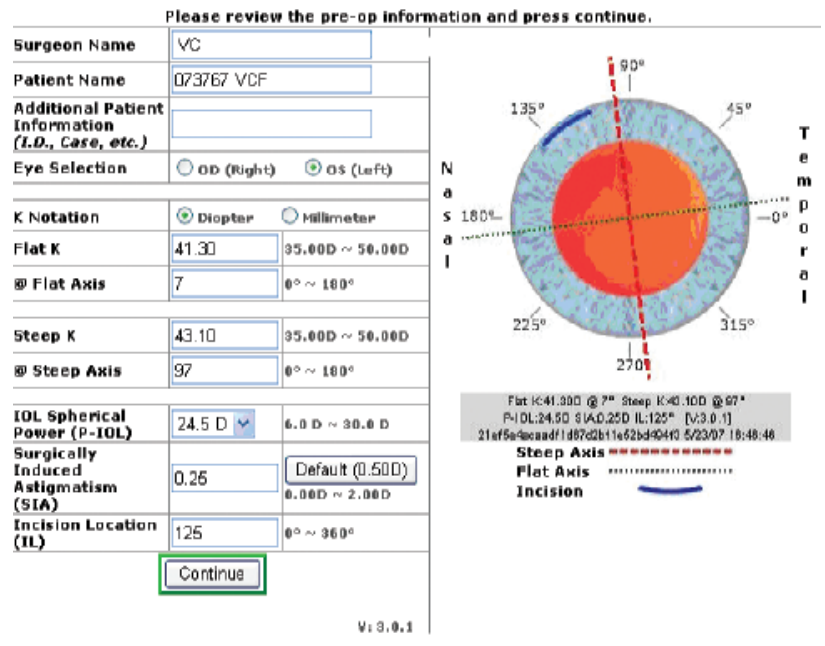

Fonte: www.acrysoftoriccalculator.com

Figura 1: Dados para o cálculo do modelo da LIO e do local do eixo do implante

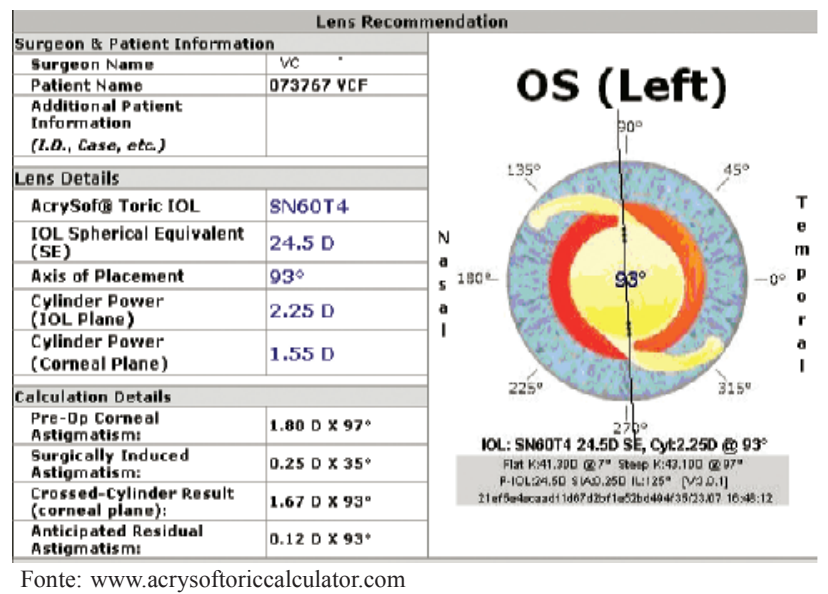

Figura 2: Resultado com modelo da LIO e local do eixo a ser implantado

zado o programa AcrySof Toric Calculator disponível em www.acrysoftoriccalculator.com, onde devem ser inseridos os seguintes dados: a ceratometria mais plana, a ceratometria mais curva, a biometria previamente calculada, a localização da incisão e o astigmatismo induzido (Figura 1).

O resultado (lens recommendation) nos orienta em relação ao modelo da LIO, a localização do eixo aonde implantar, a correção desejada e a possível refração residual (Figura 2).

$\mathrm{O}$ astigmatismo induzido pelo cirurgião foi calculado previamente por meio da análise de 10 olhos, através de um estudo comparativo da ceratometria topográfica pré e pós-operatória com seus respectivos meridianos. Esses dados foram inseridos numa tabela de análise 
Tabela 1

Análise vetorial da indução de astigmatismo incisional

\begin{tabular}{ccccccccc}
\hline & \multicolumn{3}{c}{ Pré-operatório } & \multicolumn{3}{c}{ Pós-operatório } \\
\hline & \multicolumn{3}{c}{ Meridiano } & \multicolumn{3}{c}{ Meridiano } & Meridiano & \multicolumn{2}{c}{ Meridiano } \\
\hline Olho & K1 & K1 & K2 & K2 & K1 & K1 & K2 & K2 \\
\hline E & 46.81 & 4 & 44.70 & 94 & 46.00 & 6 & 44.41 & 96 \\
E & 42.24 & 22 & 41.72 & 112 & 42.50 & 20 & 41.50 & 110 \\
D & 45.98 & 91 & 44.00 & 1 & 45.24 & 103 & 43.60 & 13 \\
D & 44.70 & 164 & 44.23 & 74 & 44.54 & 178 & 44.48 & 88 \\
E & 43.21 & 44 & 42.78 & 134 & 43.49 & 48 & 42.68 & 138 \\
E & 47.07 & 15 & 45.67 & 105 & 47.20 & 16 & 45.95 & 106 \\
D & 42.08 & 67 & 41.16 & 157 & 42.19 & 57 & 41.21 & 147 \\
E & 44.06 & 106 & 43.60 & 16 & 44.29 & 100 & 43.77 & 10 \\
D & 46.62 & 87 & 44.41 & 177 & 45.49 & 81 & 44.00 & 171 \\
E & 43.95 & 82 & 43.44 & 172 & 44.04 & 78 & 43.49 & 168 \\
\hline
\end{tabular}

- zona óptica: $6.0 \mathrm{~mm}$

- tipo óptico: biconvexa anterior

- háptico: stableforce ${ }^{\circledR}$

- comprimento: $13.0 \mathrm{~mm}$

- características especiais: dobrável, peça única

- dioptrias: $10.00-26.00$

- constante A SRK: 118.4 por imersão 118.9 IOL Master

- $\quad$ modelo: SN60T3: 1,50 = 1.03 dioptrias

SN60T4: $2,25=1.50$ dioptrias

SN60T5: $3,00=2.06$ dioptrias

- cartucho: $\mathrm{C}$

- injetor: Monarch II

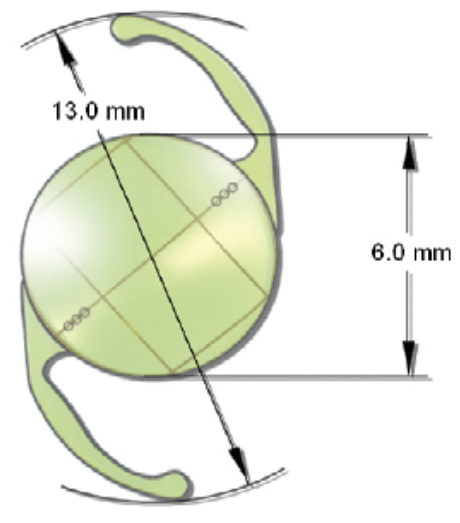

vetorial de indução de astigmatismo incisional fornecido pelo fabricante. No nosso caso o astigmatismo induzido pelo cirurgião foi de $0.25 \mathrm{DC}$ (Tabela 1 ).

A LIO utilizada neste estudo foi a SN60T - Alcon, que apresenta três (3) modelos (Tabela 2).

A lente utilizada, SN60T (Figura 3), apresenta as seguintes características:

$\mathrm{Na}$ região óptica da $\mathrm{LIO}$, próximo a base dos hápticos, existem marcas (Figura 4) que servem de guia para a implantação da LIO no eixo previamente planejado.

Segundo a fabricante, as características do háptico (Figura 5), denominado de stableforce ${ }^{\circledR}$, permitem uma boa adaptação da LIO a diferentes tamanhos de saco capsular, portanto se espera uma boa performance em relação à previsibilidade e estabilidade rotacional.
Fonte: Alcon Laboratórios do Brasil

Tabela 2

Poder de correção no plano LIO e córnea das LIOs tóricas

\begin{tabular}{cccc}
\hline Modelo & $\begin{array}{c}\text { Plano } \\
\text { LIO }\end{array}$ & $\begin{array}{c}\text { Plano } \\
\text { corneano }\end{array}$ & $\begin{array}{c}\text { Faixa de } \\
\text { correção }\end{array}$ \\
\hline 1- SN60T3 & $1.50 \mathrm{DC}$ & $1.03 \mathrm{DC}$ & $0.75-1.50 \mathrm{DC}$ \\
2- SN60T4 & $2.25 \mathrm{DC}$ & $1.55 \mathrm{DC}$ & $1.50-2.00 \mathrm{DC}$ \\
3- SN60T5 & 3.00 DC & 2.06 DC & $2.00-3.00 \mathrm{DC}$ \\
\hline
\end{tabular}

Os pacientes assinaram o termo de consentimento informado e os procedimentos foram realizados por um único cirurgião (VC), sempre com a mesma técnica de facoemulsificação e o tempo de seguimento foi de no mínimo 3 meses e máximo 6 meses sendo que o intervalo entre 
Tabela 3

Ceratometria, refração pré e pós-operatória

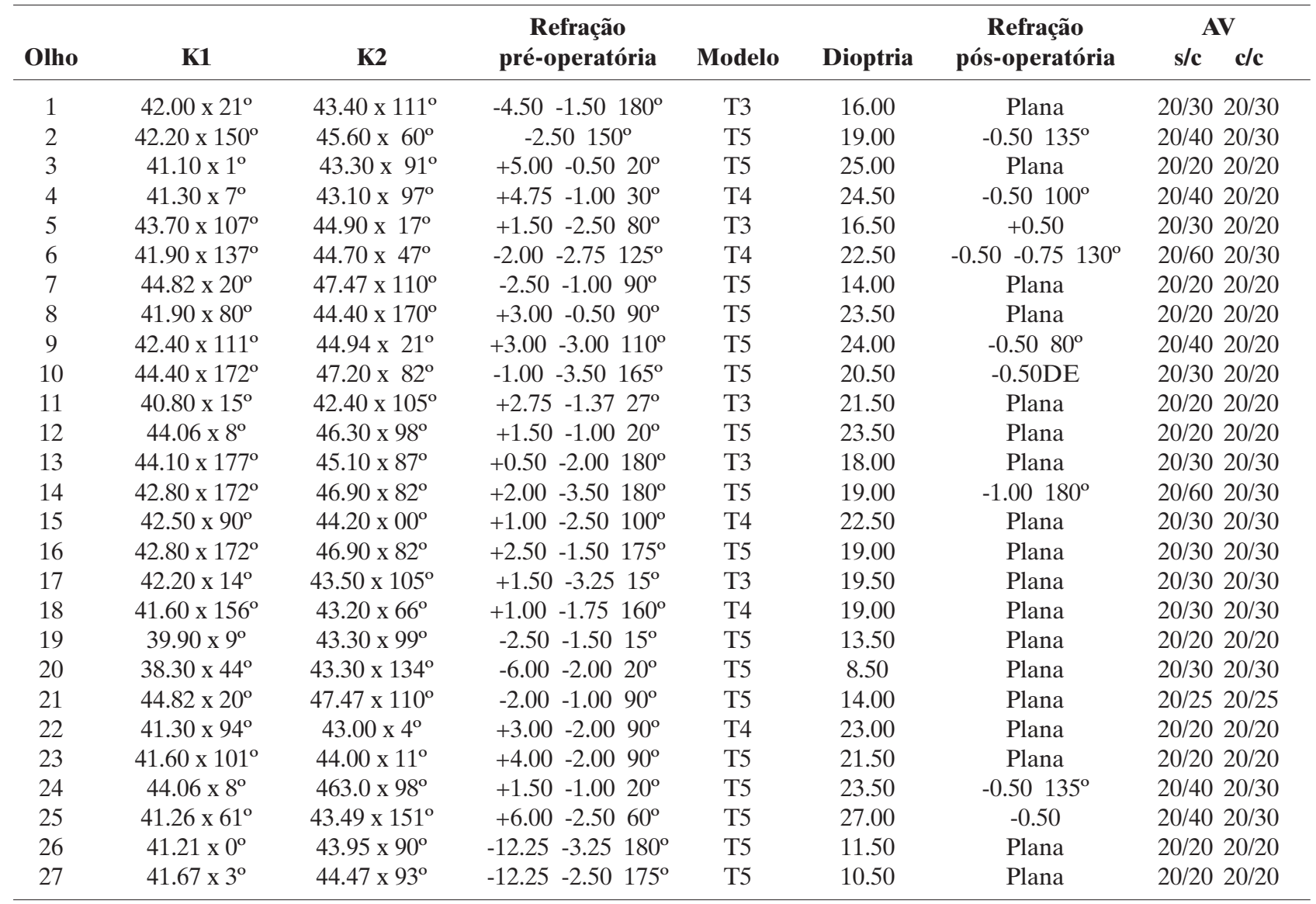

a cirurgia do primeiro e segundo olho foi de uma semana.

Prévio à cirurgia de catarata realizamos a marcação dos pontos de referência com o paciente sentado utilizando um marcador de ceratotomia radial (4 radiais) marcando $0^{\circ}, 90^{\circ}, 180^{\circ}$. Ao deitar o paciente e pronto para iniciar a cirurgia, colocamos o anel de Mendes e marcamos a $125^{\circ}$ o local da incisão e a seguir as marcas de alinhamento da LIO.

A técnica cirúrgica foi realizada através da incisão oblíqua corneana num plano de $2.75 \mathrm{~mm}$ realizada com bisturi de diamante, capsulorrexe de $\pm 5.0-5.50 \mathrm{~mm}$, técnica de stop and chop e implante da LIO com o injetor Monarch II e cartucho C.

Após o implante no saco capsular, rodamos a LIO até $\pm 20^{\circ}$ antes da posição definitiva, à qual atingimos após aspiração do material viscoelástico. Uma vez conferido o correto posicionamento da LIO, fazemos infiltração da incisão corneal.

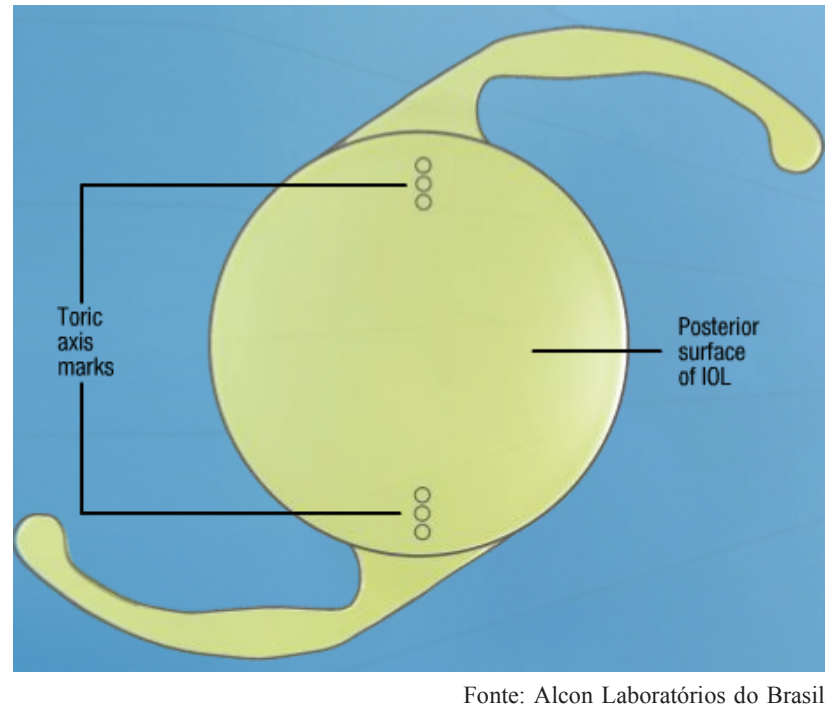

Figura 4: LIO tórica SN60T 
Tabela 4

Medida pré-operatória

\begin{tabular}{lc}
\hline Medida & Valor \\
\hline Refração esférica & \\
Média \pm dp & $-0,02 \pm 4,53$ \\
intervalo & $-12,25$ a 6,00 \\
Refração cilíndrica & \\
Média \pm dp & $-1,98 \pm 0,90$ \\
intervalo & $-3,50$ a $-0,50$ \\
Ceratometria (média $\pm d p)$ & \\
K1 & $42,25 \pm 1,53$ \\
K2 & $44,70 \pm 1,55$ \\
\hline
\end{tabular}

Tabela 6

Refração pós-operatória

\begin{tabular}{ccc}
\hline Refração & Olhos & \% \\
\hline Plana & 18 & 66,67 \\
-0.50 & 07 DC & 25,93 \\
-0.75 & $1 \mathrm{DC}$ & 3,70 \\
$\geq-1.00$ & $1 \mathrm{DC}$ & 3,70 \\
Total & 27 & 100,00 \\
& & \\
\hline
\end{tabular}

\section{Resultados}

Dos 18 pacientes estudados, $15(83,33 \%)$ eram do sexo feminino e $3(16,67 \%)$ do sexo masculino. A idade dos pacientes variou de 52 anos a 77 anos, com média de 64/83 anos.

A topografia pré-operatória e a refração pré e pós-operatória estão discriminadas na Tabela 3.

Os olhos 10 e 14 da Tabela 3 apresentam astigmatismo maior que 3.0DC. Os mesmos foram incluídos no protocolo, pois concordaram e estavam cientes de que poderiam apresentar cilíndrico residual no pós-operatório.

As medidas e desvios padrão da refração esférica e cilíndrica e ceratometria pré-operatória estão representadas na Tabela 4.

Na Tabela 5 se acompanha a distribuição dos diferentes modelos de LIO implantados.

A distribuição em grupos da refração pós-operatória se acompanha Tabela 6.

A Tabela 7 mostra a média e desvio padrão da refração esférica e cilíndrica pós-operatória.

Para verificar a diferença entre a refração cilíndrica pré-operatória e pós-operatória foi realizado o teste t pareado, obtendo-se p $<0,01$ (ou seja a nível de 1\%, ou
Tabela 5

Modelos de LIO implantada

\begin{tabular}{ccc}
\hline LIO modelo & Olhos & \% \\
\hline SN60T3 & 5 & 18,52 \\
SN60T4 & 5 & 18,52 \\
SN60T5 & 17 & 62,96 \\
Total & 27 & 100,00 \\
\hline
\end{tabular}

Tabela 7

Média e desvio padrão pós-operatória

\begin{tabular}{lcc}
\hline Medida & \multicolumn{2}{c}{ Média e desvio padrão } \\
\cline { 2 - 3 } & Pré-operatória & Pós-operatória \\
\hline Refração esférica ( D ) & $-0,02 \pm 4,53$ & $-0,04 \pm 0,19$ \\
Refração cilíndrica ( C ) & $-1,98 \pm 0,90$ & $-0,14 \pm 0,28$ \\
\hline
\end{tabular}

Tabela 8

Acuidade visual pós-operatória com e sem correção

\begin{tabular}{ccc}
\hline Olho & AV S/C & AV C/C \\
\hline 1 & $20 / 30$ & $20 / 30$ \\
2 & $20 / 40$ & $20 / 30$ \\
3 & $20 / 20$ & $20 / 20$ \\
4 & $20 / 40$ & $20 / 20$ \\
5 & $20 / 30$ & $20 / 20$ \\
6 & $20 / 60$ & $20 / 30$ \\
7 & $20 / 20$ & $20 / 20$ \\
8 & $20 / 20$ & $20 / 20$ \\
9 & $20 / 40$ & $20 / 20$ \\
10 & $20 / 30$ & $20 / 20$ \\
11 & $20 / 20$ & $20 / 20$ \\
12 & $20 / 20$ & $20 / 20$ \\
13 & $20 / 30$ & $20 / 30$ \\
14 & $20 / 60$ & $20 / 30$ \\
15 & $20 / 30$ & $20 / 30$ \\
16 & $20 / 30$ & $20 / 30$ \\
17 & $20 / 30$ & $20 / 30$ \\
18 & $20 / 30$ & $20 / 30$ \\
19 & $20 / 20$ & $20 / 20$ \\
20 & $20 / 30$ & $20 / 30$ \\
21 & $20 / 25$ & $20 / 25$ \\
22 & $20 / 20$ & $20 / 20$ \\
23 & $20 / 20$ & $20 / 20$ \\
24 & $20 / 40$ & $20 / 30$ \\
25 & $20 / 40$ & $20 / 30$ \\
26 & $20 / 20$ & $20 / 20$ \\
27 & $20 / 20$ & $20 / 20$ \\
\hline & &
\end{tabular}




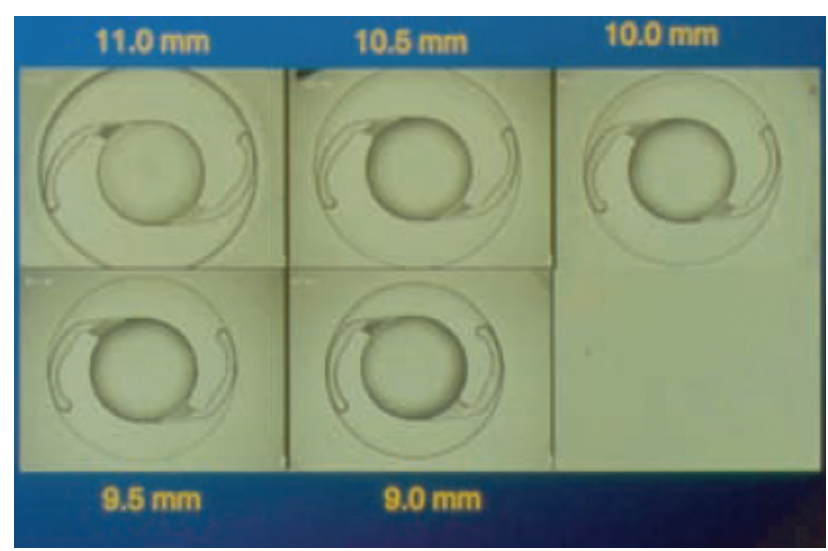

Figura 5: Stableforce ${ }^{\circledR}$

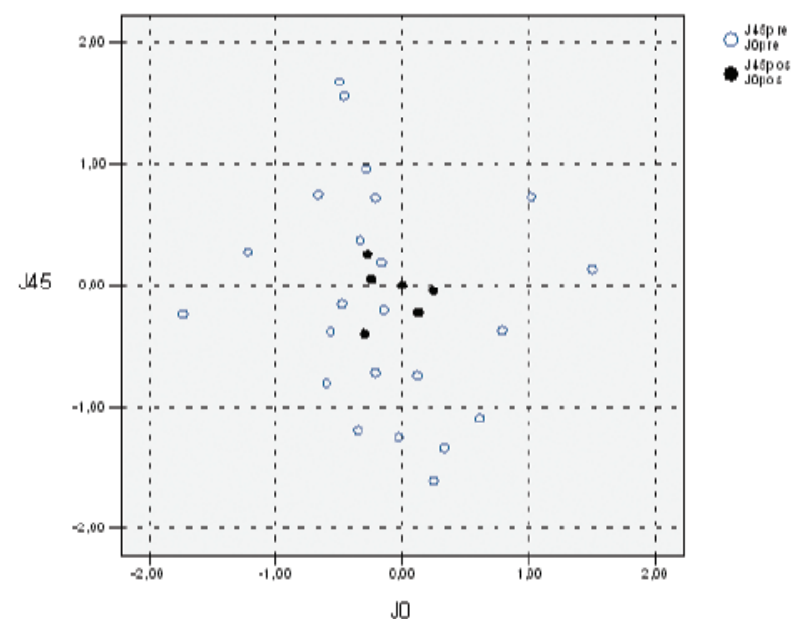

Figura 6: Distribuição refracional

Do ponto de vista da técnica cirúrgica se recomenda uma capsulotomia contínua, a mais circular possível, central com diâmetro de 5 a $5.5 \mathrm{~mm}$. Necessita de uma precisa identificação do meridiano de maior poder refrativo para o correto alinhamento da lente no saco capsular. A grande vantagem desta LIO é a reversibilidade do procedimento, pois podemos rodar a LIO ou substituí-la em caso de necessidade ${ }^{(8)}$.

Como já descrito anteriormente, a LIO tórica possui marcas na área óptica que indicam a posição do cilindro positivo que deverão se alinhar com as marcas feitas no limbo corneal.

A rotura zonular ou a evidência de desinserção do saco capsular podem contraindicar a inserção da LIO tórica, pois o bom resultado depende de uma centralização precisa da LIO.

Mendicute $^{(15)}$ em estudo de 30 olhos com implante de SN60T obteve 93,3\% dos olhos com acuidade visual não corrigida de 20/40 ou melhor, redução de 70\% do astigmatismo estando em $93.9 \%$ dentro de \pm 0.50 D. A média rotacional da LIO foi de 3,63+/- 3,11 graus com rotação menor que 10 graus em $96,7 \%$ dos olhos.

Grabow ${ }^{(16)}$ reportou 5\% de incidência de rotação maior que $30^{\circ}$ em 6 meses de seguimento em 81 casos de implante de lente 4203T da Staar Surgical Inc.

Till ${ }^{(17)}$ em 100 consecutivos casos de implante de lente intraocular tórica Staar, obteve astigmatismo refrativo residual entre $\pm 1,0$ dioptria em $75 \%$ dos olhos e entre \pm 0.50 dioptrias em $48 \%$ dos olhos.

A LIO SN60T foi testada em estudo multicêntrico prospectivo randonizado envolvendo 494 pacientes e 11 investigadores, disponível em: www.fda.gov/cdrh/pdf/ p9300145015.html e mostrou que a média de rotação foi de 4 graus; cerca de $62 \%$ dos pacientes ficaram com 
0,5 dioptrias ou menos de astigmatismo residual e a AV $\geq 20 / 40$ em $94 \%$.

No presente estudo, obtivemos acuidade visual não corrigida de 20/40 ou melhor em 92,59\% dos olhos com estabilidade refracional pós 180 dias e a previsibilidade refracional em $\pm 0.50 \mathrm{D}$ em $92,60 \%$. Observamos que há necessidade de se detectar precocemente uma possível rotação da lente, por isto, fazemos o seguimento deste procedimento no $1^{\circ} \mathrm{dia}, 7^{\circ} \mathrm{dia}, 21^{\circ} \mathrm{dia}$ pós-operatório.

A rotação, se presente, ocorre normalmente nas primeiras 24 horas. Detectada a rotação, corrigir prontamente evitando a aderência capsular.

Portanto, os três parâmetros estudados: acuidade visual $\geq 20 / 40$, estabilidade rotacional e previsibilidade refracional de $\pm 0.50 \mathrm{DC}$, fazem da SN60T uma opção válida para o tratamento do astigmatismo corneano durante a cirurgia do cristalino, tornando o paciente menos ou totalmente independente ao uso de correção para longe.

\section{Abstract}

Purpose: To present refraction results using toric intraocular lens for correcting corneal astigmatism during cataract surgery. Methods: The study comprised 27 eyes of 18 cataract patients with topographic corneal astigmatism higher than 1.0D and lower than 3.0D, submitted to cataract surgery using the phacoemulsification system, during the period of july to december 2007. Results: Non-corrected visual acuity was $\geq 20 / 40$ in $92.59 \%$ (25 eyes); post-surgical refraction was plana in $66.61 \%$ and +0.50 in $92.60 \%$. Conclusion: the use of toric IOL is a good option to neutralize corneal astigmatism between 1.0 and 3.0 diopters.

Keywords: Lenses, intraocular; Astigmatism; Cataract extraction; Phacoemulsification; Biometry/ methods; Refractive erros

\section{RefERÊNCIAS}

1. Lacava AC, Caballero JC, Medeiros OA, Centurion V. Biometria no alto míope. Rev Bras Oftalmol. 1995;54(4):61-4.

2. Centurion V, Lacava AC, Lucca ES. Facoemulsificação em pacientes portadores de catarata e alta miopia. Rev Bras Oftalmol.1998;57(11):831-5.

3. Centurion V, Lacava AC, Caballero JC, Porto RB. Lente intraocular dobrável de silicone: experiência com 2000 implantes. Rev Bras Oftalmol. 1999;58(6):419-23.

4. Caballero JC, Lacava AC, Centurion V. A biometria em olhos com catarata e óleo de silicone. Rev Bras Oftalmol. 2003;62(9):627-33.
5. Lacava AC, Carrari MJ, Centurion V. A biometria em olhos com comprimento axial médio: estudo comparativo de três fórmulas e a previsibilidade refracional. Rev Bras Oftalmol. 2006;65(3):157-61.

6. Lacava AC, Centurion V. A biometria em olhos hipermétropes: estudo comparativo de três fórmulas e a previsibilidade refracional. Rev Bras Oftalmol. 2006;65(3):162-6.

7. Centurion V, Lacava AC, Caballero JC. Lente intra-ocular multifocal difrativa apodizada: resultados. Rev Bras Oftalmol. 2007;66(6):383-7.

8. Cristóbal JA, del Buey MA, Mateo AJ. Astigmatismo y catarata. In: Centurion V, Nicoli C, Villar-Kuri J. El libro del cristalino de las Américas. São Paulo: Santos; 2007. p. 819-43.

9. Gills JP, Gayton JL. Reducing pre-existing astigmatism. In: Gills JP, editor. Cataract surgery: the state of the art. Thorofare: Slack; 1998. p. 53-66.

10. Martin RG. Management of preexisting astigmatism. In: Gills JP, Martin RG, Sanders DR, editors. Sutureless cataract surgery: an evolution toward minimaly invasive technique. Thorofare: Slack; 1992. p. 118-22.

11. Gills JP, Van der Karr M, Cherchio M. Combined toric intraocular lens implantation and relaxing incisions to reduce high preexisting astigmatism. J Cataract Refract Surg. 2002;28(9):1585-8.

12. Hoffer KJ. Biometry of 7,500 cataractous eyes. Am J Ophthalmol. 1980;90(3):360-8.

13. Hida WT, Motta AFP, Inomata DL, Jales MQM, Facio Júnior AC, José Júnior NK, Nakano CT. Incisões relaxantes limbares ou incisões no meridiano mais curvo associadas a facoemulsificação com implante de lente intra-ocular multifocal: relato de três casos. Arq Bras Oftalmol. 2008;71(2):273-7.

14. Ninn-Pedersen K, Stenevi U, Ehinger B. Cataract patients in a defined Swedish population 1986-1990. II. Preoperative observations. Acta Ophthalmol (Copenh). 1994;72(1):10-5.

15. Mendicute J, Irigoyen C, Aramberri J, Ondarra A, MontésMicó R. Foldable toric intraocular lens for astigmatism correction in cataract patients. J Cataract Refract Surg. 2008;34(4):601-7.

16. Grabow HB. Toric intraocular lens report. Ann Ophthalmol.1997;29(3):161-3.

17. Till JS, Yoder PR Jr, Wilcox TK, Spielman JL. Toric intraocular lens implantation: 100 consecutive cases. J Cataract Refract Surg. 2002;28(2):295-301.

18. Gills JP, Van der Karr MA. Correcting high astigmatism with piggyback toric intraocular lens implantation. J Cataract Refract Surg. 2002;28(3):547-9. Comment in: J Cataract Refract Surg. 2002;28(3):379-80.

19. Frohn A, Dick HB, Thiel HJ. Implantation of a toric poly(methyl methacrylate) intraocular lens to correct high astigmatism. J Cataract Refract Surg. 1999;25(12):1675-8.

\section{ENDEREÇO PARA CORRESPONDÊNCIA: \\ Virgilio Centurion \\ Av. Ibirapuera, 624 - Ibirapuera \\ CEP 04028-000 - São Paulo - SP \\ E-mail: centurion@imo.com.br}

